\title{
OPEN A nomogram combining plasma fibrinogen and systemic immune-inflammation index predicts survival in patients with resectable gastric cancer
}

\author{
Pan-Xing Wang, Hai-Jiang Wang, Jia-Huang Liu, Guang-Lin Qiu, Jing Lu, Lin Fan, \\ Xin-Hua Liao \& Xiang-Ming Che $\mathbb{1}^{\mathbb{}}$
}

Hyperfibrinogenemia and cancer-associated systemic inflammatory response are strongly associated with cancer progression and prognosis. We aimed to develop a novel prognostic score (F-SII score) on the basis of preoperative fibrinogen (F) and systemic immunoinflammatory index (SII), and evaluate its predictive value in patients with resectable gastric cancer (GC). Patients diagnosed with GC between January 2012 and December 2016 were reviewed. The F-SII score was 2 for patients with a high fibrinogen level $(\geq 3.37 \mathrm{~g} / \mathrm{L}$ ) and a high SII $(\geq 372.8)$, whereas that for patients with one or neither was 1 or 0 , respectively. A high F-SIl score was significantly associated with older patient age, a high ASA score, large tumor size, large proportion of perineural invasion, and late TNM stage. Multivariate analysis indicated that the F-SII score, histological grade, and TNM stage were independent factors for overall survival (OS). The Harrell's concordance index (C-index) of a nomogram based on the F-SII score and several clinicopathological manifestations was 0.72 , which showed a better predictive ability for OS than the TNM stage alone (0.68). In conclusion, preoperative F-SII may serve as a useful predictive factor for $\mathrm{OS}$ and refine outcome prediction for patients with resectable GC combined with traditional clinicopathological analysis.

Gastric cancer (GC) is the fifth most common malignancy and the third leading cause of cancer-associated mortality worldwide ${ }^{1}$. Despite the significant advances in therapeutic methods, including surgical techniques and adjuvant therapy, the prognosis of GC has not been substantially improved ${ }^{2}$. Therefore, identifying independent prognostic factors is of utmost importance for optimizing personalized treatment.

Inflammation plays a vital role in the tumor microenvironment and is related to the promotion, progression, invasion, and metastasis of tumors ${ }^{3}$. Pretreatment serum-based inflammatory biomarkers, such as the neutrophilto-lymphocyte ratio (NLR) ${ }^{4}$, platelet-to-lymphocyte ratio (PLR) ${ }^{5}$, and lymphocyte-to-monocyte ratio (LMR) ${ }^{6}$, was used to predict the prognosis of various tumors. Recently, the systemic immune-inflammation index (SII), a newly emerging prognostic score as an integrated expression of neutrophils, lymphocytes, and platelets, is deemed to provide better prognostic information on patients with hepatocellular carcinoma ${ }^{7}$, pancreatic cancer ${ }^{8}$, germ-cell tumors ${ }^{9}$, and gastric cancer ${ }^{10}$. In addition to inflammatory biomarkers, fibrinogen is a $340-\mathrm{kDa}$ glycoprotein, synthesized as an acute-phase reactant glycoprotein by hepatocytes and has an important role in the coagulation process ${ }^{11}$. Mounting evidence has reported that hyperfibrinogenemia is related to tumor progression, metastasis, and prognosis in patients with gastric cancer ${ }^{12,13}$. High SII and elevated fibrinogen independently predict a worse prognosis of GC. However, the evidence is still limited regarding the joint association between both conditions and the prognosis in patients with GC.

Therefore, in this study, we developed a novel prognostic score combining the fibrinogen $(\mathrm{F})$ and systemic immune-inflammation index (F-SII) score, and evaluated the association between the F-SII score and the prognosis in patients with resectable GC. Moreover, a nomogram combining the F-SII score with TNM stage, and histological grade, was established to predict 3- and 5-yr overall survival (OS) for patients with resectable GC. 


\section{Results}

Patient characteristics. In the present study, a total of 608 patients were included. The median patient age was 61 years (range 25-86 years). The median follow-up period was 56.0 months (interquartile range, 41-71 months). The 1-yr, 3-yr, and 5-yr OS rates for the present study were $85.9 \%, 58.3 \%$, and $48.0 \%$, respectively. The baseline characteristics of the patients are summarized in Table 1.

Associations of the plasma fibrinogen level, SII, and F-SII score. The univariate analysis showed that the fibrinogen level and SII were associated with OS. Age, ASA score, tumor location, tumor size, histological grade, perineural invasion, and TNM stage also had a significant effect on OS (Table 2). According to our multivariate analysis, the fibrinogen level and SII were independent factors for prognosis (HR, 1.509; 95\% CI, 1.181-1.929; $P=0.001 ; \mathrm{HR}, 1.452 ; 95 \% \mathrm{CI}, 1.128-1.868 ; P=0.004$, respectively). In addition, well or moderately differentiated tumors and stage I disease were associated with good prognosis in GC (Table 2).

Patients were classified into two independent groups based on the cutoff thresholds of fibrinogen and SII (low $<3.37 \mathrm{~g} / \mathrm{L}$ or high $\geq 3.37 \mathrm{~g} / \mathrm{L}$ and low $<372.8$ or high $\geq 372.8$, respectively) for subsequent analyses. It is showed that decreased plasma fibrinogen and SII were both associated with shorter OS (both $P<0.001$ ) (Fig. 1A,B). To further investigate the effect of the plasma fibrinogen level and SII on patient prognosis, we divided the patients into four groups based on the cutoff thresholds of fibrinogen and SII. Kaplan-Meier analysis indicated obvious differences between the four subgroups $(P<0.001$, Fig. 1C). There was no significant difference in subgroups of either plasma fibrinogen $\geq 3.37 \mathrm{~g} / \mathrm{L}$ or SII $\geq 372.8(P>0.05$, Fig. $1 \mathrm{C})$. Therefore, we combined the two subgroups. Patients were divided into three F-SII score subgroups based on the following criteria: score 2, both a high fibrinogen level $(\geq 3.37 \mathrm{~g} / \mathrm{L})$ and a high SII $(\geq 372.8)$; score 1, either a high fibrinogen level or a high SII; and score 0, both a low fibrinogen level $(<3.37 \mathrm{~g} / \mathrm{L})$ and a low SII $(<372.8)$.

The F-SII score independently predicts OS. The univariate analysis showed that the F-SII score had a significant effect on OS $(P<0.001)$. The results of the multivariate analysis indicated that the F-SII score, histological grade, and TNM stage were independent prognostic factors of OS in GC patients $($ all $P<0.05)$ (Table 2). Kaplan-Meier analysis showed that a high F-SII score was associated with short OS $(P<0.05$, Fig. 1D). To further analyze the F-SII score's performance in patients with different TNM stages and adjuvant chemotherapy strategies, we conducted a subgroup analysis. When stratified by TNM stage, there was no significant difference in 5 -yr OS between the three groups of patients with stage I GC $(P=0.144$; Fig. $2 \mathrm{~A})$. However, the prognostic value of the F-SII score was maintained for stages II $(P=0.002$; Fig. $2 \mathrm{~B})$, I-II $(P<0.001$; Fig. $2 \mathrm{C})$ and III $(P<0.001$; Fig. 2D). The F-SII also stratified OS irrespective of adjuvant chemotherapy administration $(P<0.05 ;$ Fig. 3A,B).

Associations of the plasma fibrinogen level, SII, and F-SII score with clinicopathological characteristics. The associations of the plasma fibrinogen level and SII with clinicopathological characteristics are shown in Table 3. Elevated plasma fibrinogen levels and a high SII were associated with older age at surgery $(P<0.001$ and $P=0.021)$, a high ASA score $(P=0.006$ and $P=0.015)$, tumor size $\geq 5 \mathrm{~cm}$ (both $P<0.001)$, and a late TNM stage (both $P<0.001$ ). Moreover, we assessed the association between the F-SII score and clinicopathological factors (Table 3). A high F-SII score was associated with older patient age $(P<0.001)$, a high ASA score $(P=0.002)$, large tumor size $(P<0.001)$, a large proportion of perineural invasion $(P=0.033)$, and late TNM stage $(P<0.001)$ (Table 3$)$.

Predictive nomogram for OS. To evaluate the predictive value of the F-SII score, we constructed a nomogram that integrated the independent prognostic factors consisting of TNM stage, histological grade, and F-SII score (Fig. 4A). In this nomogram, each factor was ascribed a weighted point total that indicated a survival prognosis. For internal validation, the calibration curve suggested that the 3 - and 5-yr survival rates predicted by the nomogram were consistent with the actual survival rates (Fig. 4B,C). The Harrell's concordance index (C-index) of the nomogram was 0.72 , which showed a better predictive ability for OS than the TNM stage (C-index 0.68 ) and F-SII (C-index 0.62). The areas under the 3-yr and 5-yr ROC curves of the nomogram were 0.797 and 0.80 , respectively (Fig. $5 \mathrm{~A}, \mathrm{~B}$ ). Therefore, combined with the above results, the nomogram is superior to the TNM staging system in predicting the OS of patients with GC.

\section{Discussion}

In this study, we confirmed that the preoperative plasma fibrinogen and SII were independent prognostic factors in patients with resectable GC. Moreover, the F-SII score, a newly proposed cumulative score, remained an independent prognostic factor in the multivariate analysis. In addition, its prognostic significance was maintained in the subgroup analysis of patients diagnosed with TNM stages I-II or stage III, as well as patients who did or did not receive adjuvant chemotherapy. We found that a high F-SII score was also associated with older age at surgery, a high ASA score, a large tumor size, the presence of perineural invasion, and a late TNM stage. Then, we developed a prognostic nomogram that included the TNM stage, histological grade, and F-SII score and predicted OS with an accuracy of 0.72 . Thus, the F-SII score as an easy and inexpensive indicator might provide important prognostic information to help clinicians estimate the patient outcome by combining with conventional clinicopathological analysis. To the best of our knowledge, this is the first study to determine the clinical value of the F-SII score in patients with resectable GC.

It was reported that plasma fibrinogen is synthesized as an acute-phase reactant glycoprotein by hepatocytes. Several studies have reported the mechanisms of hyperfibrinogenemia in various tumors ${ }^{13-16}$. In patients with lung cancer, interleukin- 6 produced by tumor cells stimulates the secretion of fibrinogen ${ }^{15}$. More importantly, 


\begin{tabular}{|c|c|c|}
\hline Characteristics & No & $\%$ \\
\hline \multicolumn{3}{|l|}{ Age (years) } \\
\hline$\geq 60$ & 354 & 58.2 \\
\hline$<60$ & 254 & 41.8 \\
\hline \multicolumn{3}{|l|}{ Sex } \\
\hline Male & 461 & 75.8 \\
\hline Female & 147 & 24.2 \\
\hline \multicolumn{3}{|l|}{ BMI $\left(\mathrm{kg} / \mathrm{m}^{2}\right)$} \\
\hline$\geq 24$ & 160 & 26.3 \\
\hline$<24$ & 448 & 73.7 \\
\hline \multicolumn{3}{|l|}{ ASA score } \\
\hline 1 & 40 & 6.6 \\
\hline 2 & 446 & 73.4 \\
\hline 3 & 122 & 20.1 \\
\hline \multicolumn{3}{|l|}{ Tumor location } \\
\hline Upper & 207 & 34.0 \\
\hline Middle & 124 & 20.4 \\
\hline Lower & 277 & 45.6 \\
\hline \multicolumn{3}{|l|}{ Tumor size $(\mathbf{c m})$} \\
\hline$\geq 5$ & 267 & 43.9 \\
\hline$<5$ & 341 & 56.1 \\
\hline \multicolumn{3}{|l|}{ Histological grade } \\
\hline Well or moderately differentiated & 202 & 33.2 \\
\hline Poorly or not differentiated & 406 & 66.8 \\
\hline \multicolumn{3}{|l|}{ Vascular invasion } \\
\hline Yes & 68 & 11.2 \\
\hline No & 540 & 88.8 \\
\hline \multicolumn{3}{|l|}{ Perineural invasion } \\
\hline Yes & 217 & 35.7 \\
\hline No & 391 & 64.3 \\
\hline \multicolumn{3}{|l|}{ Lymphatic invasion } \\
\hline Yes & 65 & 10.7 \\
\hline No & 543 & 89.3 \\
\hline \multicolumn{3}{|l|}{ Pathological tumor stage } \\
\hline $\mathrm{T} 1$ & 98 & 16.1 \\
\hline $\mathrm{T} 2$ & 36 & 5.9 \\
\hline T3 & 47 & 7.7 \\
\hline $\mathrm{T} 4$ & 427 & 70.2 \\
\hline \multicolumn{3}{|l|}{ Pathological lymph node stage } \\
\hline No & 225 & 37 \\
\hline $\mathrm{N} 1$ & 105 & 17.3 \\
\hline N2 & 117 & 19.2 \\
\hline N3 & 161 & 26.5 \\
\hline \multicolumn{3}{|l|}{ TNM stage } \\
\hline I & 115 & 18.9 \\
\hline II & 141 & 23.2 \\
\hline III & 352 & 57.9 \\
\hline \multicolumn{3}{|l|}{ Adjuvant chemotherapy } \\
\hline Yes & 325 & 53.5 \\
\hline No & 283 & 46.5 \\
\hline \multicolumn{3}{|l|}{ Fibrinogen level (g/L) } \\
\hline$\geq 3.37$ & 258 & 42.4 \\
\hline$<3.37$ & 350 & 57.6 \\
\hline \multicolumn{3}{|l|}{ SII } \\
\hline$\geq 372.8$ & 328 & 53.9 \\
\hline$<372.8$ & 280 & 46.1 \\
\hline \multicolumn{3}{|l|}{ F-SII score } \\
\hline Continued & & \\
\hline
\end{tabular}




\begin{tabular}{|l|l|l|}
\hline Characteristics & No & $\%$ \\
\hline 0 & 208 & 34.2 \\
\hline 1 & 214 & 35.2 \\
\hline 2 & 186 & 30.6 \\
\hline
\end{tabular}

Table 1. Patient and tumour characteristics. BMI Body Mass Index, ASA score American Society of Anesthesiologists score, SII Systemic immune-inflammation index, F-SII Fibrinogen and systemic immuneinflammation index.

\begin{tabular}{|c|c|c|c|c|c|c|}
\hline \multirow[b]{2}{*}{ Characteristics } & \multirow{2}{*}{$\begin{array}{l}\text { Univariate analysis } \\
\text { HR }(95 \% \mathrm{CI})\end{array}$} & \multirow[b]{2}{*}{$P$-values } & \multirow{2}{*}{\begin{tabular}{|l|} 
Multivariate analysis $^{\mathrm{a}}$ \\
HR $(95 \% \mathrm{CI})$ \\
\end{tabular}} & \multirow[b]{2}{*}{$P$-values } & \multirow{2}{*}{$\begin{array}{l}\text { Multivariate analysis } \\
\text { HR }(95 \% \mathrm{CI})\end{array}$} & \multirow[b]{2}{*}{$P$-values } \\
\hline & & & & & & \\
\hline Age ( $\geq 60$ vs. $<60$ years) & $1.269(1.006-1.602)$ & 0.045 & $1.052(0.821-1.348)$ & 0.689 & $1.054(0.823-1.349)$ & 0.678 \\
\hline Sex (Female vs. Male) & $0.815(0.615-1.073)$ & 0.145 & & & & \\
\hline BMI ( $\geq 24$ vs. $<24$ kg/m2) & $0.895(0.689-1.162)$ & 0.405 & & & & \\
\hline ASA score & & 0.009 & & 0.272 & & 0.282 \\
\hline 2 vs. 1 & $1.118(0.690-1.813)$ & 0.650 & $0.993(0.608-1.623)$ & 0.979 & $0.992(0.607-1.620)$ & 0.973 \\
\hline 3 vs. 1 & $1.663(0.992-2.786)$ & 0.054 & $1.240(0.728-2.114)$ & 0.428 & $1.235(0.725-2.106)$ & 0.437 \\
\hline Tumor location & & 0.004 & & 0.227 & & 0.224 \\
\hline Middle vs. upper & $0.825(0.606-1.124)$ & 0.224 & $0.984(0.713-1.358)$ & 0.923 & $0.986(0.714-1.362)$ & 0.933 \\
\hline Lower vs. upper & $0.651(0.505-0.840)$ & 0.001 & $0.810(0.624-1.051)$ & 0.112 & $0.809(0.624-1.050)$ & 0.112 \\
\hline Tumor size ( $\geq 5$ vs. $<5 \mathrm{~cm}$ ) & $2.187(1.737-2.753)$ & $<0.001$ & $1.251(0.983-1.591)$ & 0.069 & $1.253(0.985-1.594)$ & 0.066 \\
\hline $\begin{array}{l}\text { Histological grade: Well or moderately differentiated vs. Poorly or } \\
\text { not differentiated }\end{array}$ & $0.498(0.382-0.651)$ & $<0.001$ & $0.721(0.546-0.952)$ & 0.021 & $0.721(0.546-0.952)$ & 0.021 \\
\hline Vascular invasion (Yes vs. No) & $1.309(0.938-1.828)$ & 0.113 & & & & \\
\hline Perineural invasion (Yes vs. No) & $1.714(1.362-2.157)$ & $<0.001$ & $1.034(0.815-1.312)$ & 0.781 & $1.033(0.813-1.311)$ & 0.792 \\
\hline Lymphatic invasion (Yes vs. No) & $1.159(0.817-1.643)$ & 0.408 & & & & \\
\hline TNM stage & & $<0.001$ & & $<0.001$ & & $<0.001$ \\
\hline II vs. I & $4.254(2.212-8.183)$ & $<0.001$ & $3.125(1.602-6.094)$ & 0.001 & $3.119(1.599-6.084)$ & 0.001 \\
\hline III vs. I & $11.485(6.267-21.048)$ & $<0.001$ & $7.619(4.050-14.332)$ & $<0.001$ & $7.614(4.047-14.322)$ & $<0.001$ \\
\hline Adjuvant chemotherapy (Yes vs. No) & $1.033(0.823-1.297)$ & 0.780 & & & & \\
\hline Fibrinogen level ( $\geq 3.37$ vs. $<3.37 \mathrm{~g} / \mathrm{L})$ & $2.097(1.668-2.636)$ & $<0.001$ & $1.509(1.181-1.929)$ & 0.001 & & \\
\hline SII $(\geq 372.8$ vs. $<372.8)$ & $2.013(1.584-2.558)$ & $<0.001$ & $1.452(1.128-1.868)$ & 0.004 & & \\
\hline F-SII score & & $<0.001$ & & & & $<0.001$ \\
\hline 1 vs. 0 & $1.493(1.094-2.036)$ & 0.012 & & & $1.505(1.097-2.066)$ & 0.011 \\
\hline 2 vs. 0 & $2.656(1.979-3.564)$ & $<0.001$ & & & $2.201(1.612-3.004)$ & $<0.001$ \\
\hline
\end{tabular}

Table 2. Univariate and multivariate Cox regression analyses for overall survival in patients with gastric cancer. BMI Body Mass Index, ASA score American Society of Anesthesiologists score, SII Systemic immune-inflammation index, F-SII score Fibrinogen and systemic immune-inflammation index score. adjustment for all variables listed in the table, except for sex, BMI, vascular invasion, lymphatic invasion, chemotherapy, and F-SII score. ${ }^{\mathrm{b}}$ Adjustment for all variables listed in the table, except for sex, BMI, vascular invasion, lymphatic invasion, chemotherapy, fibrinogen level, and SII.

fibrinogen, which is synthesized by cancer cells, promotes the proliferation of fibroblast growth factor- $2^{16}$. Finally, plasma fibrinogen promotes tumor cell growth and angiogenesis by interacting with fibroblast growth factor- 2 and vascular endothelial growth factor ${ }^{16,17}$. On the other hand, since Virchow originally made a link between cancer and inflammation in the nineteenth century, a growing body of evidence ${ }^{3,18}$ has suggested that the levels of inflammatory markers play a vital role in tumor progression and metastasis. Hu et al. ${ }^{7}$ reported that a high SII was related to liver cirrhosis, a large tumor size, low tumor differentiation, early recurrence, high circulating tumor cell levels, and a poor prognosis in patients with hepatocellular carcinoma. Moreover, Wang et al. ${ }^{10}$ found that high SII was associated with old age at surgery, poor Borrmann classification, a large tumor size, advanced tumor invasion, lymph node metastasis, distant metastasis, advanced TNM stage, a high CEA level, and poor outcome in patients with gastric cancer. It was reported that a high SII was also connected with sex, the hemoglobin level, and a poor prognosis in patients with small-cell lung cancer ${ }^{19}$. In the present study, we showed that the plasma fibrinogen level and SII are independent prognostic factors of OS in GC patients. Therefore, we created the F-SII score consisting of the plasma fibrinogen level and SII. 
A

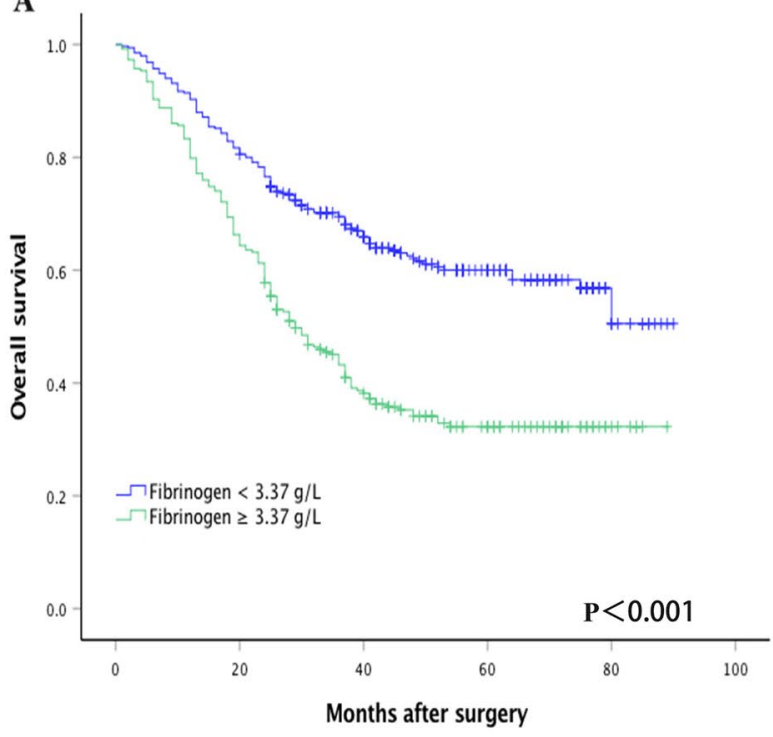

C

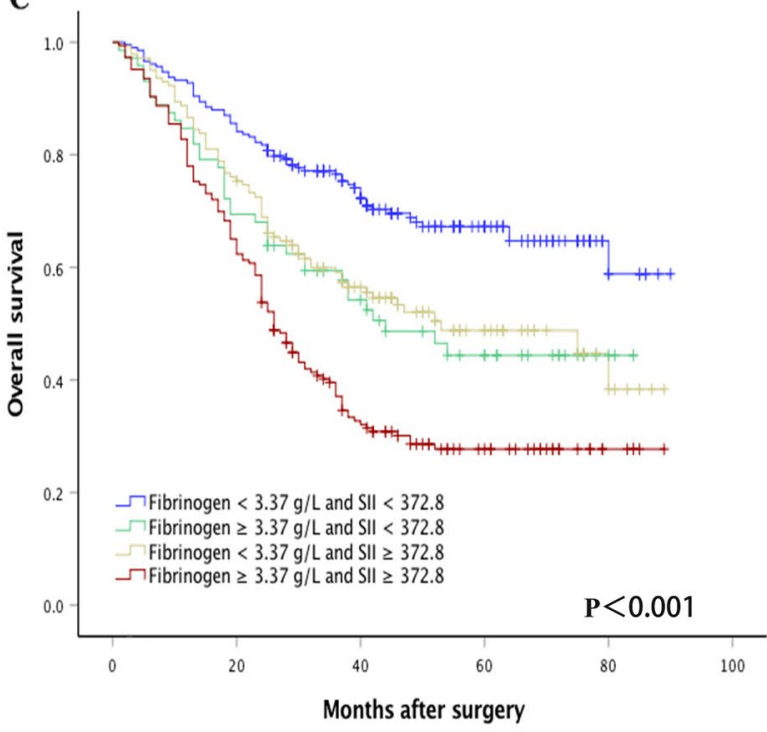

B

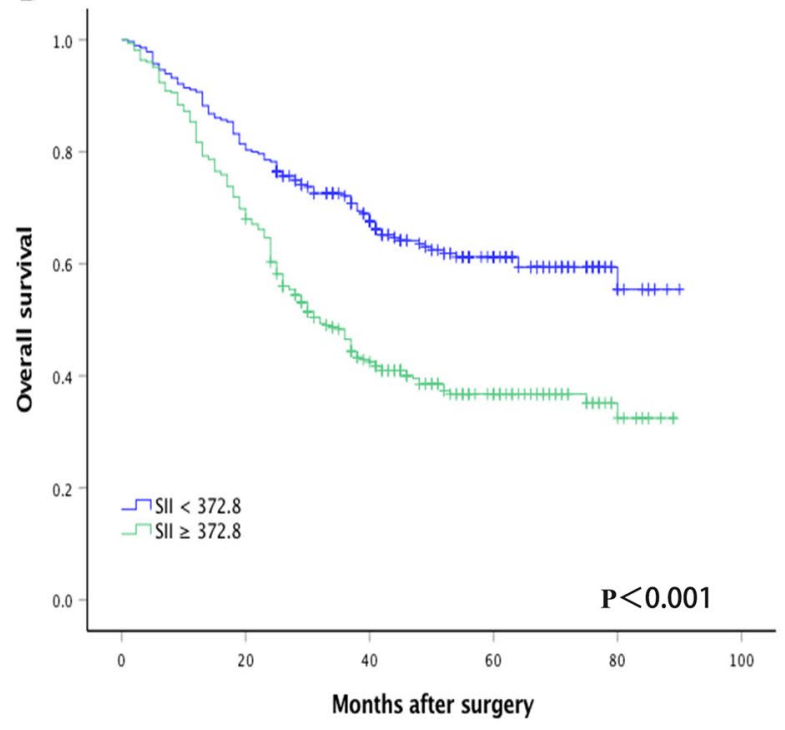

D

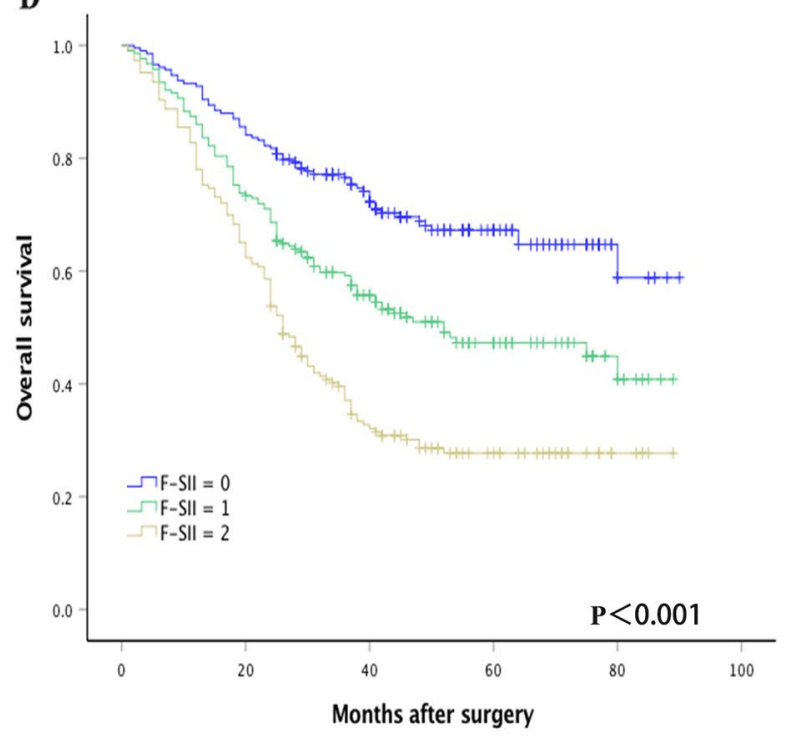

Figure 1. Kaplan-Meier analysis for OS of GC patients according to preoperative plasma fibrinogen level and the systemic immune-inflammation index (SII). Kaplan-Meier analysis for OS according to (A) preoperative plasma fibrinogen level, (B) preoperative SII, (C) combination of preoperative plasma fibrinogen and SII, and (D) F-SII score.

In agreement with previous findings, we demonstrated that both a high fibrinogen level and a high SII (F-SII score 2) are related to advanced tumor stage and a poor prognosis. In contrast, decreased levels of both (F-SII score 0 ) are related to early tumor stage and a favorable prognosis. Furthermore, a high F-SII score was associated with aggressive tumor biological phenotypes, such as large tumor size, the presence of perineural invasion, and advanced tumor stage. Combined with the above results, the complex interaction between an elevated systemic inflammatory response and tumor progression was partially revealed. Of note, its prognostic significance was still maintained in the subgroup analysis of patients diagnosed with TNM stages I-II or stage III, as well as patients who did or did not receive adjuvant chemotherapy, suggesting that the F-SII score might provide additional prognostic information as a complement to the complete clinicopathological predictive models. As a result, the F-SII score could be an accurate prognostic indicator.

At present, the nomogram fulfills a necessary role in personalisation of oncological treatments by integrating diverse prognostic and determinant variables to generate the probability of a clinical event ${ }^{20}$. In our study, we developed a nomogram that includes the preoperative TNM stage, histological grade, and F-SII score to improve outcome prediction in GC patients after surgery. We found that the nomogram showed more accurate predictive 
A

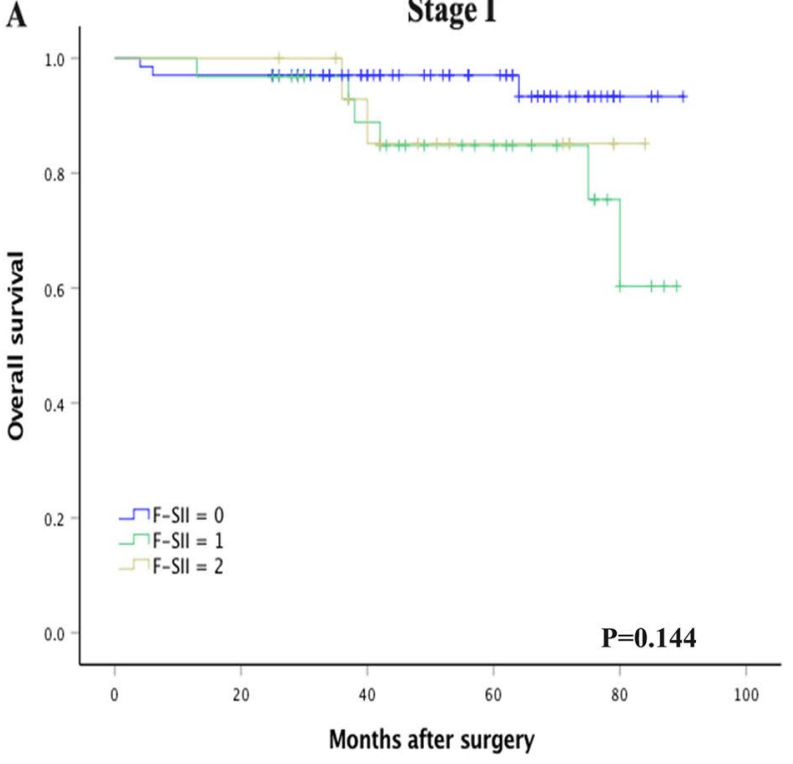

C

Stage I-II

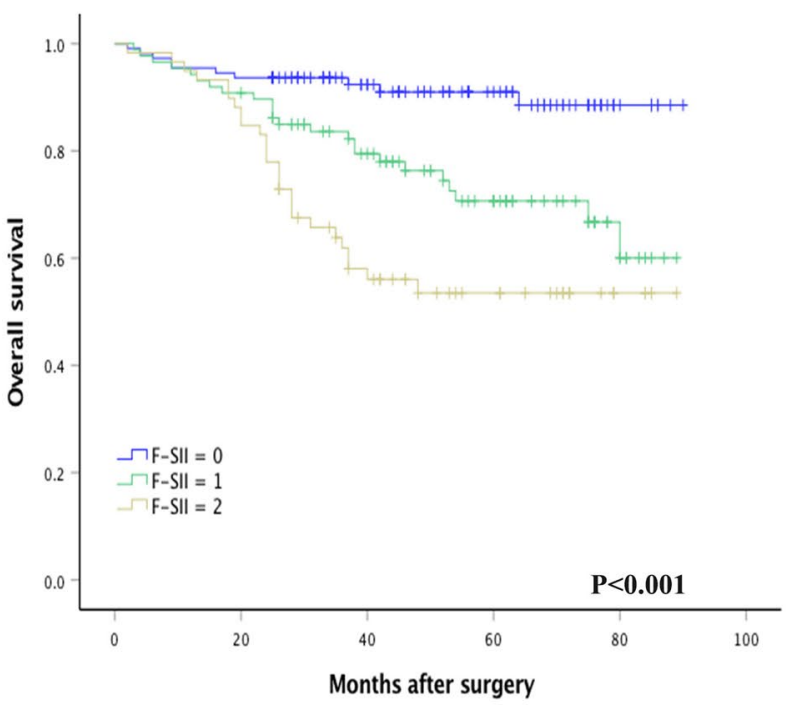

B

B

Stage II

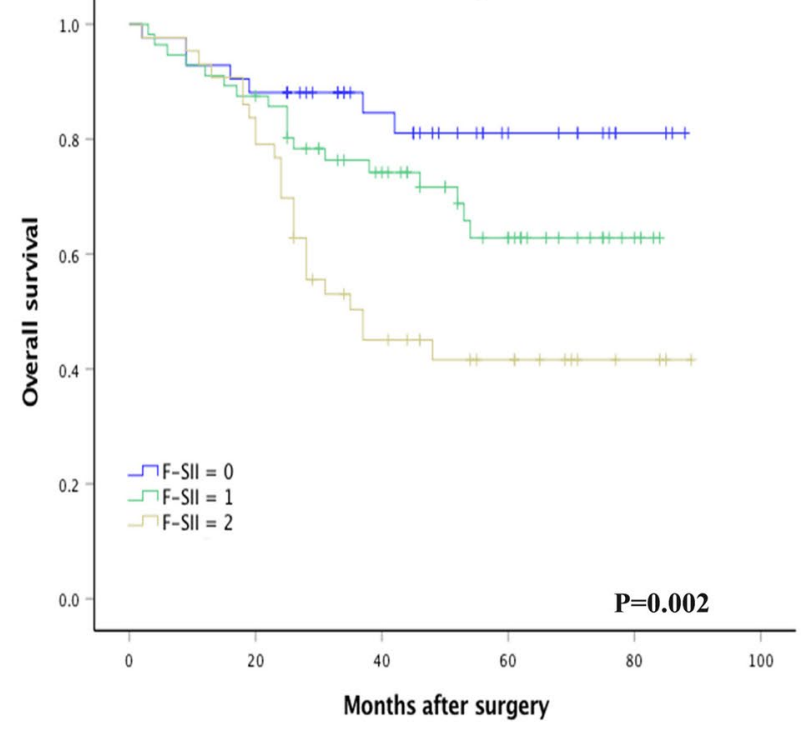

D

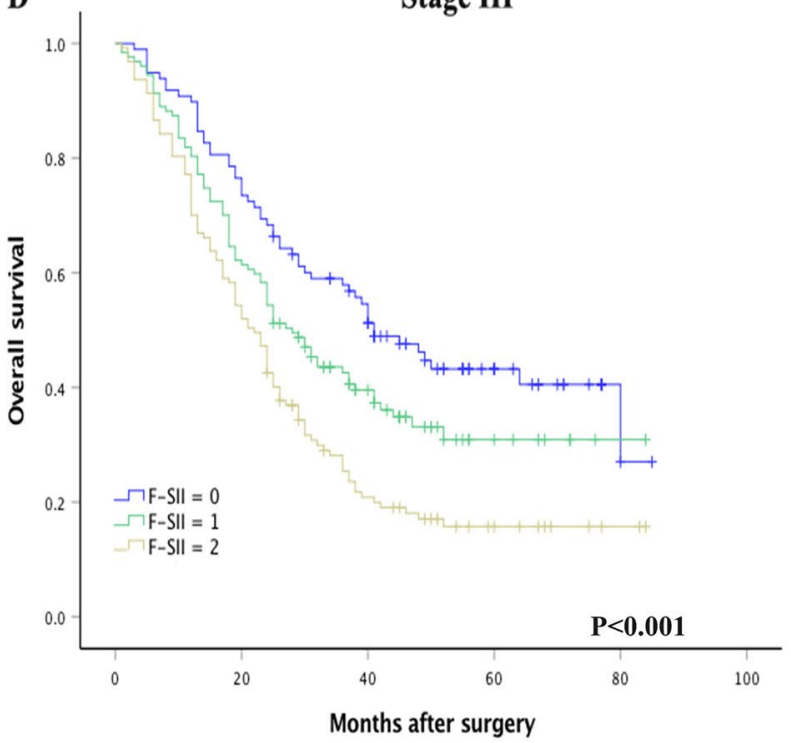

Figure 2. Kaplan-Meier analysis of OS of GC patients at each TNM stage according to the F-SII score. (A) Association of the F-SII score with the OS of patients with stage I GC. (B) Association of the F-SII score with the OS of patients with stage II GC. (C) Association of the F-SII score with the OS of patients with stage I-II GC. (D) Association of the F-SII score with the OS of patients with stage III GC.

ability than the TNM stage alone. In addition, the F-SII score can be considered a supplement to physical examinations, such as cross-sectional imaging, endoscopic ultrasonography, and endoscopy, to refine risk stratification in patients with gastric cancer before and after treatment.

The strength of our study is that F-SII score measurements were based on standard laboratory tests of plasma fibrinogen and platelet, neutrophil, and lymphocyte counts, which are routinely used in clinical practice. Nevertheless, our study has certain limitations. First, due to the retrospective nature of the study and the lack of external validation, the prognostic significance of the F-SII score in GC patients remains to be examined prospectively in other populations and larger studies in the future. Second, hematological cell counts may be affected by several factors, though we limited some of the possible confounders. Third, we lacked follow-up information for diseasefree survival (DFS), and the application of other survival outcomes may strengthen our findings. 
A

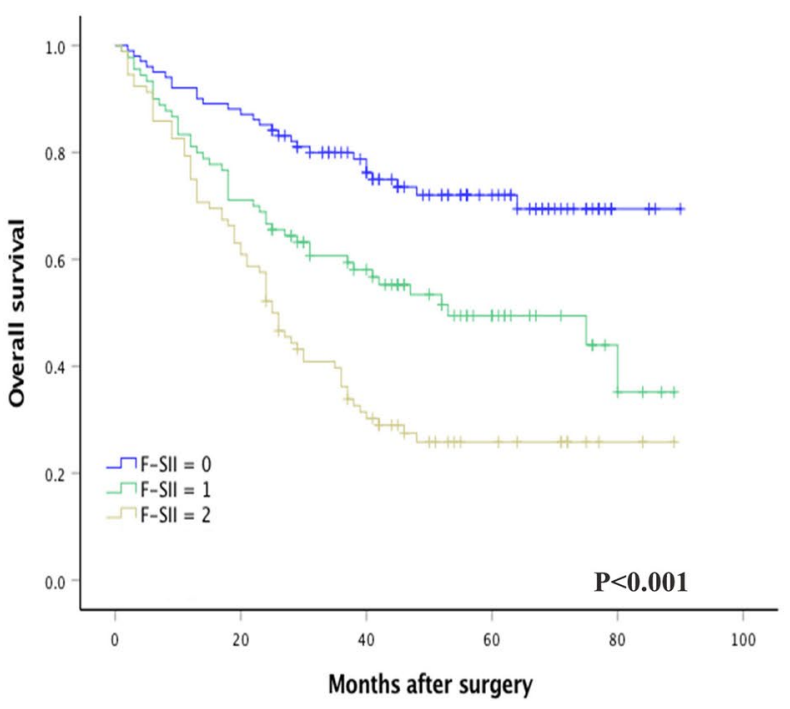

B

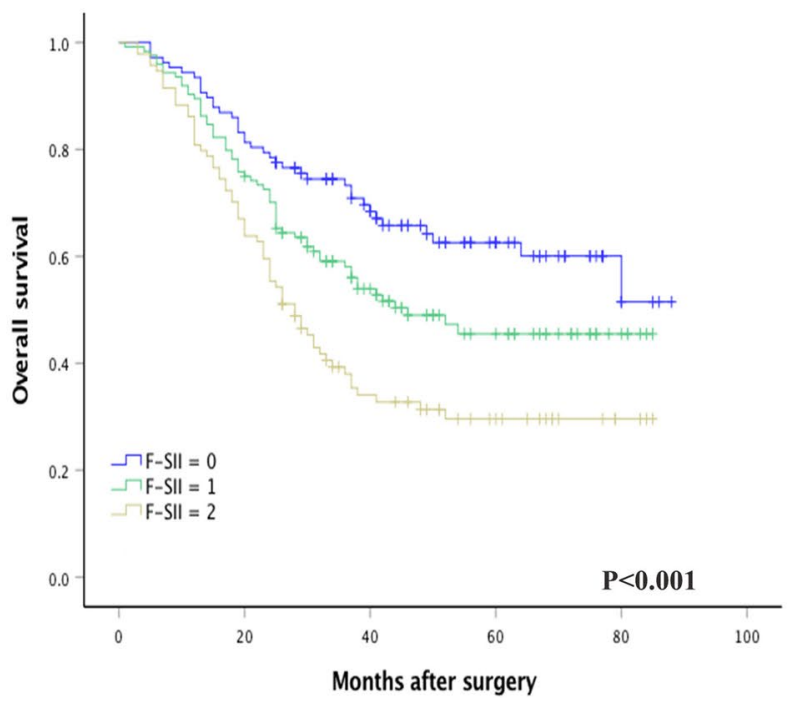

Figure 3. (A) Association of the F-SII score with the OS in the surgery alone group. (B) Association of the F-SII score with the OS in the adjuvant chemotherapy group.

\section{Conclusion}

In conclusion, we created a novel and convenient prognostic score named the F-SII score, which was revealed an independent predictor of survival in patients with resectable GC. The F-SII score may be a useful clinical biomarker for identifying patients at high prognostic risk and planning individualized treatment strategies for GC patients.

\section{Methods}

Patient characteristics. We collected data from 608 consecutive patients with resectable gastric adenocarcinoma who were treated between January 2012 and December 2016 at the Department of General Surgery, The First Affiliated Hospital of Xian Jiaotong University. All patients provided informed consent prior to study participation. This study was approved by the Institutional Review Board of the First Affiliated Hospital of Xian Jiaotong University and conducted in compliance with the principles of the Declaration of Helsinki for medical research involving humans. The inclusion criteria of this study were as follows: (1) gastric adenocarcinoma confirmed histopathologically, (2) complete medical records, and (3) underwent radical gastrectomy. The exclusion criteria of this study were as follows: (1) other malignancies, (2) neoadjuvant chemotherapy, (3) metastatic disease, (4) autoimmune or other inflammatory diseases, (5) perioperative mortality, (6) hematological disease, (7) intravenous or arterial embolization within 3 months and (8) continuous anticoagulant therapy. We gathered the following clinical, pathologic, and laboratory data of the patients: age, sex, BMI, American Society of Anesthesiologists (ASA) score, tumor location, tumor size, histological grade, vascular invasion, perineural invasion, lymphatic invasion, TNM stage ${ }^{21}$, adjuvant chemotherapy, fibrinogen, and SII. In our hospital, 5-fluorouracilbased adjuvant chemotherapy is routinely delivered to patients with advanced $\mathrm{GC}^{22}$.

The SII and F-SII score. Preoperative plasma fibrinogen, lymphocyte, neutrophil, and platelet count levels were examined in samples obtained before breakfast within 7 days prior to surger ${ }^{23,24}$. As defined previously, the SII was defined as follows: SII = platelet count $\times$ neutrophil count/lymphocyte count ${ }^{7}$. The optimal cut-off values for plasma fibrinogen (low $<3.37$; high $\geq 3.37 \mathrm{~g} / \mathrm{L}$ ) and SII (low $<372.8$; high $\geq 372.8$ ) were obtained through ROC curves ${ }^{25}$. The F-SII score was established based on the combination of different plasma fibrinogen levels and SII values.

Follow-up. Enrolled patients were prospectively followed-up until June 2019. Patients were routinely followed up every 3 months for the first 2 years after treatment and every 6 months thereafter. Patients evaluations included laboratory tests, a physical examination, multislice computed tomography, and other examinations. OS was defined as the time from the date of surgery to death from any cause or the last follow-up. 


\begin{tabular}{|c|c|c|c|c|c|c|c|c|c|c|}
\hline & \multicolumn{3}{|c|}{ Fibrinogen level $(\mathrm{g} / \mathrm{L})$} & \multicolumn{3}{|l|}{ SII } & \multicolumn{4}{|c|}{ F-SII score } \\
\hline & $<3.37$ & $\geq 3.37$ & $P$-values & $<372.8$ & $\geq 372.8$ & $P$-values & 0 & 1 & 2 & $P$-values \\
\hline Characteristics & $\mathrm{n}=350$ & $\mathrm{n}=258$ & & $\mathrm{n}=280$ & $\mathrm{n}=328$ & & $\mathrm{n}=208$ & $\mathrm{n}=214$ & $\mathrm{n}=186$ & \\
\hline Age (years) & & & $<0.001$ & & & 0.021 & & & & $<0.001$ \\
\hline$\geq 60$ & 179 & 175 & & 149 & 205 & & 101 & 126 & 127 & \\
\hline$<60$ & 171 & 83 & & 131 & 123 & & 107 & 88 & 59 & \\
\hline Sex & & & 0.144 & & & 0.165 & & & & 0.904 \\
\hline Male & 273 & 188 & & 205 & 256 & & 159 & 160 & 142 & \\
\hline Female & 77 & 70 & & 75 & 72 & & 49 & 54 & 44 & \\
\hline BMI $\left(\mathrm{kg} / \mathrm{m}^{2}\right)$ & & & 0.065 & & & 0.124 & & & & 0.114 \\
\hline$\geq 24$ & 102 & 58 & & 82 & 78 & & 64 & 56 & 40 & \\
\hline$<24$ & 248 & 200 & & 198 & 250 & & 144 & 158 & 146 & \\
\hline ASA score & & & 0.006 & & & 0.015 & & & & 0.002 \\
\hline 1 & 22 & 18 & & 19 & 21 & & 14 & 13 & 13 & \\
\hline 2 & 273 & 173 & & 219 & 227 & & 171 & 150 & 125 & \\
\hline 3 & 55 & 67 & & 42 & 80 & & 23 & 51 & 48 & \\
\hline Tumor location & & & 0.559 & & & 0.792 & & & & 0.707 \\
\hline Upper & 115 & 92 & & 96 & 111 & & 67 & 77 & 63 & \\
\hline Middle & 69 & 55 & & 60 & 64 & & 46 & 37 & 41 & \\
\hline Lower & 166 & 111 & & 124 & 153 & & 95 & 100 & 82 & \\
\hline Tumor size $(\mathrm{cm})$ & & & $<0.001$ & & & $<0.001$ & & & & $<0.001$ \\
\hline$\geq 5$ & 117 & 150 & & 97 & 170 & & 60 & 94 & 113 & \\
\hline$<5$ & 233 & 108 & & 183 & 158 & & 148 & 120 & 73 & \\
\hline Histological grade & & & 0.179 & & & 0.168 & & & & 0.091 \\
\hline Well or moderately different & 124 & 78 & & 101 & 101 & & 81 & 63 & 58 & \\
\hline Poorly or not differentiated & 226 & 180 & & 179 & 227 & & 127 & 151 & 128 & \\
\hline Vascular invasion & & & 0.281 & & & 0.55 & & & & 0.586 \\
\hline Yes & 35 & 33 & & 29 & 39 & & 20 & 24 & 24 & \\
\hline No & 315 & 225 & & 251 & 289 & & 188 & 190 & 162 & \\
\hline Perineural invasion & & & 0.236 & & & 0.063 & & & & 0.033 \\
\hline Yes & 118 & 99 & & 89 & 128 & & 60 & 87 & 70 & \\
\hline No & 232 & 159 & & 191 & 200 & & 148 & 127 & 116 & \\
\hline Lymphatic invasion & & & 0.707 & & & 0.611 & & & & 0.605 \\
\hline Yes & 36 & 29 & & 28 & 37 & & 19 & 26 & 20 & \\
\hline No & 314 & 229 & & 252 & 291 & & 189 & 188 & 166 & \\
\hline TNM stage & & & $<0.001$ & & & $<0.001$ & & & & $<0.001$ \\
\hline I & 89 & 26 & & 78 & 37 & & 68 & 31 & 16 & \\
\hline II & 80 & 61 & & 60 & 81 & & 42 & 56 & 43 & \\
\hline III & 181 & 171 & & 142 & 210 & & 98 & 127 & 127 & \\
\hline Adjuvant chemotherapy & & & 0.753 & & & 0.913 & & & & 0.258 \\
\hline Yes & 189 & 136 & & 149 & 176 & & 107 & 124 & 94 & \\
\hline No & 161 & 122 & & 131 & 152 & & 101 & 90 & 92 & \\
\hline
\end{tabular}

Table 3. Associations of Fibrinogen, SII, and F-SII score with clinicopathological characteristics. BMI Body Mass Index, ASA score American Society of Anesthesiologists score, SII Systemic immune-inflammation index, F-SII Fibrinogen and systemic immune-inflammation index.

Statistical analysis. Statistical analyses were performed using SPSS software (version 25.0; SPSS Inc., Chicago, IL, USA) and R version 3.6.1 software (http://www.r-project.org/). Extension packages, including "survival", "rms", "foreign", and "survivalROC" were also used. Chi-square tests were performed to analyze categorical variables. Kaplan-Meier survival curves were generated, and the log-rank test was performed to compare survival rates. The best cutoff points of plasma fibrinogen and SII were determined using the Youden index and 
A

Points

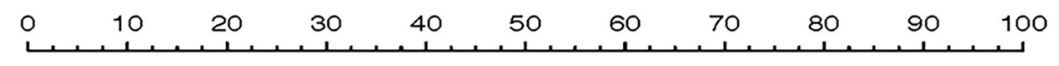

TNM

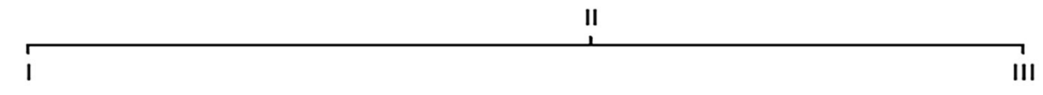

Grade

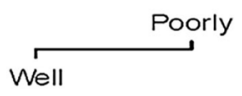

FSII

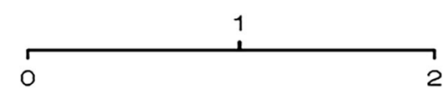

Total Points

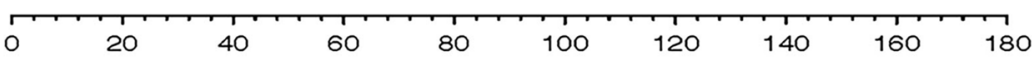

3-Year Survival

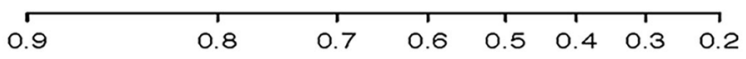

5-Year survival

0.9

0.8

$\begin{array}{lllllll}0.7 & 0.6 & 0.5 & 0.4 & 0.3 & 0.2 & 0.1\end{array}$

B

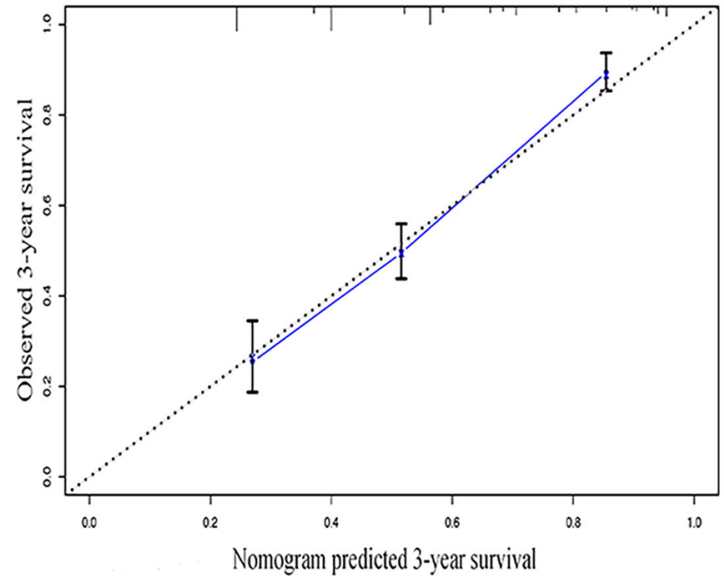

C

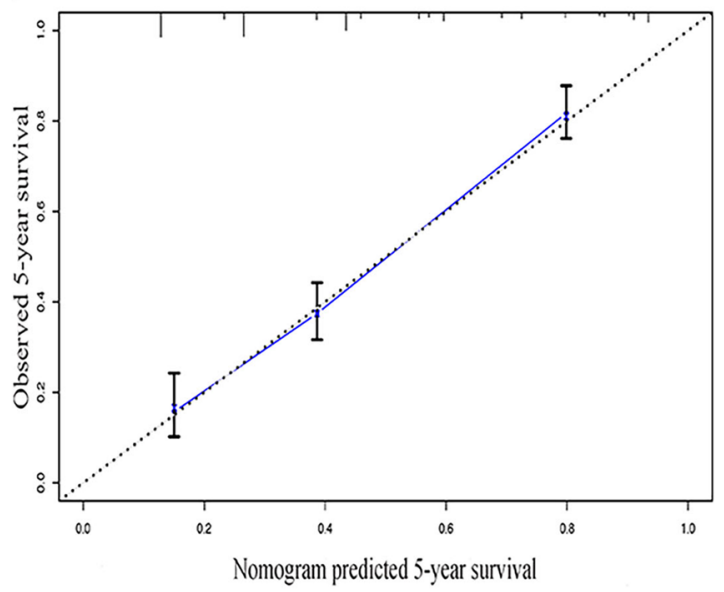

Figure 4. Nomogram for predicting 3- and 5-year OS of GC patients after surgery. (A) Nomogram for predicting 3- and 5-year OS of GC patients after surgery. Calibration plot of the nomogram for (B) 3-year and (C) 5-year survival. The dashed line represents the performance of an ideal nomogram. The blue line indicates the performance of the proposed nomogram. Blue circles are sub-cohorts of the data set; $\mathrm{X}$ is the bootstrapped corrected estimate of nomogram with 200 resamples. Vertical bars represent $95 \%$ CI. It seems that the nomogram predicts accurately 3 - and 5-year OS.

ROC curves. Multivariate analysis using a Cox proportional hazards regression model was used based on variables with a $P$-value of $<0.05$ from the univariate analysis. The nomogram was plotted based on the results of the multivariate analysis. The model's predictive accuracy was estimated by the C-index ${ }^{26}$ and ROC curve analysis. The calibration plots were applied to verify the performance characteristics of the predictive nomogram. The significance level for all statistical tests was set at 0.05 , and all tests were 2 -sided. 
A

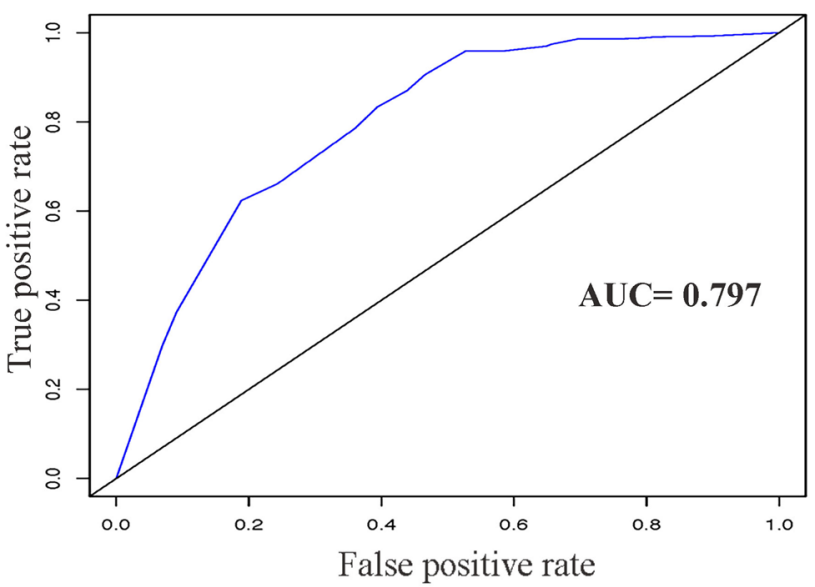

B

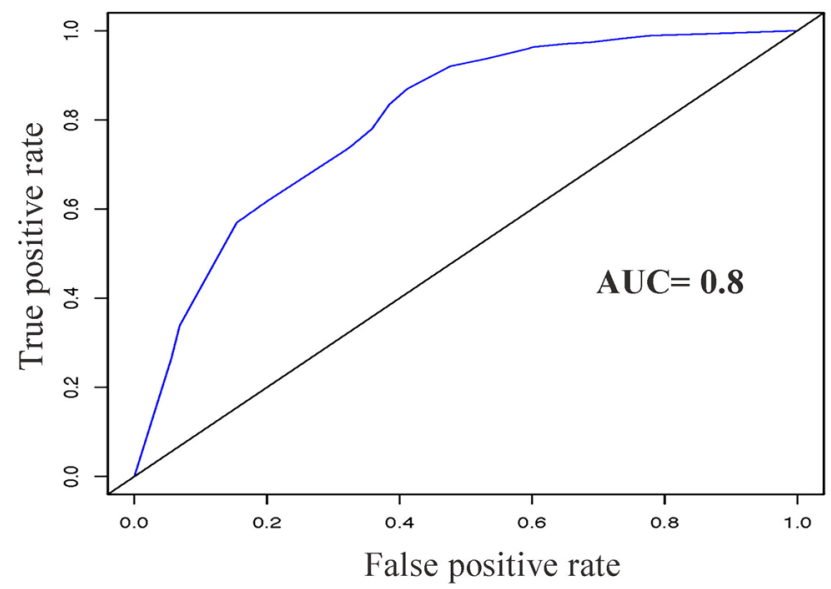

Figure 5. Time-dependent receiver operating characteristic curve analysis for the sensitivity and specificity of the nomograms. Receiver operating characteristic of the nomogram for (A) 3-yr survival and (B) 5-yr survival.

\section{Data availability}

The datasets generated and/or analysed during the current study are available from the corresponding author on reasonable request.

Received: 21 October 2020; Accepted: 29 April 2021

Published online: 13 May 2021

\section{References}

1. Ferlay, J. et al. Cancer incidence and mortality worldwide: sources, methods and major patterns in GLOBOCAN 2012. Int. J. Cancer 136, E359-386 (2015).

2. Kurokawa, Y. et al. Bursectomy versus omentectomy alone for resectable gastric cancer (JCOG1001): a phase 3, open-label, randomised controlled trial. Lancet Gastroenterol. Hepatol. 3, 460-468 (2018).

3. Mantovani, A., Allavena, P., Sica, A. \& Balkwill, F. Cancer-related inflammation. Nature 454, 436-444 (2008).

4. Piciucchi, M. et al. The neutrophil/lymphocyte ratio at diagnosis is significantly associated with survival in metastatic pancreatic cancer patients. Int. J. Mol. Sci. 18, 730 (2017).

5. Kim, E. Y. et al. The platelet-to-lymphocyte ratio versus neutrophil-to-lymphocyte ratio: which is better as a prognostic factor in gastric cancer?. Ann. Surg. Oncol. 22, 4363-4370 (2015).

6. Chan, J. et al. The lymphocyte-to-monocyte ratio is a superior predictor of overall survival in comparison to established biomarkers of resectable colorectal cancer. Ann. Surg. 265, 539-546 (2017).

7. Hu, B. et al. Systemic immune-inflammation index predicts prognosis of patients after curative resection for hepatocellular carcinoma. Clin. Cancer Res. 20, 6212-6222 (2014).

8. Aziz, M. et al. The systemic-immune-inflammation index independently predicts survival and recurrence in resectable pancreatic cancer and its prognostic value depends on bilirubin levels: a retrospective multicenter cohort study. Ann. Surg. 270, 139-146 (2019).

9. Chovanec, M. et al. Systemic immune-inflammation index in germ-cell tumours. Br. J. Cancer 118, 831-838 (2018).

10. Wang, K. et al. Prognostic value of systemic immune-inflammation index in patients with gastric cancer. Chin. J. Cancer 36, 75 (2017).

11. Arigami, T. et al. Combined fibrinogen concentration and neutrophil-lymphocyte ratio as a prognostic marker of gastric cancer. Oncol. Lett. 11, 1537-1544 (2016).

12. Yamashita, H., Kitayama, J. \& Nagawa, H. Hyperfibrinogenemia is a useful predictor for lymphatic metastasis in human gastric cancer. Jpn. J. Clin. Oncol. 35, 595-600 (2005).

13. Yamashita, H., Kitayama, J., Kanno, N., Yatomi, Y. \& Nagawa, H. Hyperfibrinogenemia is associated with lymphatic as well as hematogenous metastasis and worse clinical outcome in T2 gastric cancer. BMC Cancer 6, 147 (2006).

14. Yamamoto, M. et al. Usefulness of preoperative plasma fibrinogen versus other prognostic markers for predicting gastric cancer recurrence. World. J. Surg. 40, 1904-1909 (2016).

15. Yamaguchi, T. et al. Involvement of lnterleukin-6 in the elevation of plasma fibrinogen levels in lung cancer patients. Jpn. J. Clin. Oncol. 28, 740-744 (1998).

16. Sahni, A., Simpson-Haidaris, P. J., Sahni, S., Vaday, G. \& Francis, C. Fibrinogen synthesized by cancer cells augments the proliferative effect of FGF-2. J. Thromb. Haemost. 6, 176-183 (2008).

17. Sahni, A. \& Francis, C. Vascular endothelial growth factor binds to fibrinogen and fibrin and stimulates endothelial cell proliferation. Blood 96, 3772-3778 (2000).

18. Balkwill, F. \& Mantovani, A. Inflammation and cancer: back to Virchow?. Lancet 357, 539-545 (2001).

19. Hong, X. et al. Systemic immune-inflammation index, based on platelet counts and neutrophil-lymphocyte ratio, is useful for predicting prognosis in small cell lung cancer. Tohoku J. Exp. Med. 236, 297-304 (2015).

20. Kattan, M. Nomograms. Introduction. Semin. Urol. Oncol. 20, 79-81 (2002).

21. Edge, S. \& Compton, C. The American Joint Committee on Cancer: the 7th edition of the AJCC cancer staging manual and the future of TNM. Ann. Surg. Oncol. 17, 1471-1474 (2010).

22. Bang, Y. et al. Adjuvant capecitabine and oxaliplatin for gastric cancer after D2 gastrectomy (CLASSIC): a phase 3 open-label, randomised controlled trial. Lancet 379, 315-321 (2012).

23. Jagadesham, V., Lagarde, S., Immanuel, A. \& Griffin, S. Systemic inflammatory markers and outcome in patients with locally advanced adenocarcinoma of the oesophagus and gastro-oesophageal junction. Br. J. Surg. 104, 401-407 (2017). 
24. Wu, Z. et al. Cumulative score based on preoperative fibrinogen and pre-albumin could predict long-term survival for patients with resectable gastric cancer. J. Cancer 10, 6244-6251 (2019).

25. Lu, J. et al. CRP/prealbumin, a novel inflammatory index for predicting recurrence after radical resection in gastric cancer patients: post hoc analysis of a randomized phase III trial. Gastric Cancer 22, 536-545 (2019).

26. Harrell, F., Lee, K. \& Mark, D. Multivariable prognostic models: issues in developing models, evaluating assumptions and adequacy, and measuring and reducing errors. Stat. Med. 15, 361-387 (1996).

\section{Acknowledgements}

The authors thank American Journal Experts for providing language editing. This study was supported by grants from the National Natural Science Foundation of China (81472245) and the Fundamental Research Funds of the First Affiliated Hospital of Xi'an Jiaotong University (No. 2017QN-01).

\section{Author contributions}

X.M.C. and P.X.W. designed the study; H.J.W., J.H.L., G.L.Q., and J.L collected the data; P.X.W. and L.F. interpreted the results; P.X.W. and X.H.L. prepared the manuscript; All authors approved the final version of the manuscript.

\section{Competing interests}

The authors declare no competing interests.

\section{Additional information}

Correspondence and requests for materials should be addressed to X.-M.C.

Reprints and permissions information is available at www.nature.com/reprints.

Publisher's note Springer Nature remains neutral with regard to jurisdictional claims in published maps and institutional affiliations.

(c) (i) Open Access This article is licensed under a Creative Commons Attribution 4.0 International License, which permits use, sharing, adaptation, distribution and reproduction in any medium or format, as long as you give appropriate credit to the original author(s) and the source, provide a link to the Creative Commons licence, and indicate if changes were made. The images or other third party material in this article are included in the article's Creative Commons licence, unless indicated otherwise in a credit line to the material. If material is not included in the article's Creative Commons licence and your intended use is not permitted by statutory regulation or exceeds the permitted use, you will need to obtain permission directly from the copyright holder. To view a copy of this licence, visit http://creativecommons.org/licenses/by/4.0/.

(C) The Author(s) 2021 